\title{
New material for anti-wear corrosion-resistant coating of agro industrial complex machinery parts
}

\author{
D.N. Okhlupin ${ }^{1}, A . V$. Korolev $^{2, *}$, and I.V. Sinev ${ }^{3}$ \\ ${ }^{1}$ TechnoTherm-Saratov LLC, 410040 Saratov, Russia \\ ${ }^{2}$ The Saratov State Technical University named after Yu.A. Gagarin, 410040 Saratov, Russia \\ ${ }^{3}$ The Saratov State University named after N.G. Chernyshevsky, 410040 Saratov, Russia
}

\begin{abstract}
The article presents the results of an experimental study of the corrosion resistance of the ta-C double coating of parts by PVD method with intermediate and final surface polishing with a discrete tool. The influence of regime factors on the results of polishing the ta-C coating with a discrete tool is shown, and optimal processing modes are proposed. Tests of samples in a salt bath showed that the double ta$\mathrm{C}$ coating by PVD method with intermediate and final polishing ensures the absence of corrosion and coating peeling
\end{abstract}

\section{Introduction}

Since the development of the agro-industrial complex is carried out in the direction of increasing the reliability and durability of the equipment used, it is important to protect the surfaces of parts of this equipment from corrosion and mechanical wear. One of the most effective methods of protecting parts from abrasive wear in conditions of high environmental pollution is application of a high-strength diamond-like coating (DLC) on the surface of mating parts. DLC has unique properties, including high hardness, increased thermal conductivity, low coefficient of friction and high wear resistance. The disadvantages of DLC coatings include their porosity and, as a result, low corrosion resistance, which limits the use of these coatings in the agro-industrial complex. To eliminate this drawback, TechnoTherm-Saratov LLC has developed a technology for DLC application, which provides reliable parts protection from corrosion.

\section{Materials and methods}

The essence of the technology is as follows. Before coating, the surface of the part is thoroughly ultrasonic cleaned from various oxide films and contaminants. The diamondlike ta-C coating is applied in the DREVA 600 unit using the PVD method - a vacuum method by physically application of vapor-phase coating. To ensure the necessary adhesion,

\footnotetext{
*Corresponding author: science7@bk.ru
} 
an adhesive titanium layer is pre-applied to the surface of the part using the PVD method. The unit has a laser chamber in which solid graphite is converted to a gaseous state by laser. Then, using a dense plasma flow created by a hollow cathode and using arc vacuum evaporation sources, the vaporous graphite is converted to an excited ionized state and, under the action of a bias voltage, it is deposited on the surface of the adhesive layer of the part, turning into a diamond-like coating.

The disadvantage of this method is that the PVD method does not provide a uniform dense structure, since there are always microscopic particles of graphite and other defects on the surface of the coating, through which moisture can penetrate. Therefore, the applied coating is polished, the surface is thoroughly cleaned again, and then the part is placed again in the DREVA 600 unit, where another layer of ta-C diamond-like coating is applied. After this coating acquires not only high hardness and ability to resist wear, but also high corrosion resistance.

Until recently, the most complex in this process was the coating polishing operation. Due to the high hardness of the coating, the existing methods of grinding and finishing [14] did not provide the required surface roughness, which should be Ra 0.04 and at which the graphite particle and all other coating particles with poor adhesion are removed. Therefore, they are replaced by other methods, the most promising of which is the method of thermochemical treatment [5-8].

The thermochemical treatment method uses one of the "weak" properties of diamond - the ability to dissolve in transition metals in the presence of high temperature. The essence of methods for diamond coatings treatment based on the method of thermochemical treatment consists in the friction of the diamond coating with a counterbody made in the form of a disk made of a transition metal-iron, nickel, titanium, and others [9-10]. As a result of friction, the temperature necessary for graphitizing the diamond and removing the rough top layer of the coating is created. The process of thermochemical treatment is based on the phenomenon of catalytic gasification of diamonds in a hydrogen environment and proceeds in four stages: the transition of carbon atoms from the diamond to the surface layer of the catalyst metal; the transfer of carbon atoms in the metal volume; the transition of carbon dissolved in the metal to the gas phase; and the removal of gaseous carbon-containing compounds from the reaction zone. The disadvantages of these methods are the inability to process a coating of small thickness due to the high removal of the allowance and low productivity due to the multi-stage process.

In the development of the chemical-thermal method of diamond coatings treatment TechnoTherm-Saratov LLC, a mechanical-chemical-thermal method is proposed. The essence of this method is that the surface treatment is carried out with a discrete tool in the form of a metal brush. This creates conditions for the simultaneous implementation of two processes of impact on the treated surface mechanical and chemical-thermal. Mechanical destruction of the tops of the microroughness of the treated surface occurs from the impact of the tool fiber. Chemical and thermal treatment is carried out under the influence of high temperature resulting from the friction of the metal tool-catalyst with the surface to be treated. Thus, the effectiveness of the mechanical-chemical-thermal method is ensured by the fact that it is based on the use of two "weak" properties of diamond at once, its brittleness and the ability to dissolve in transition materials under certain conditions.

\section{Results and discussion}

The part "Prizma" produced by the Altai Precision Products Plant JSC was used as samples for experimental studies. This part is used in the fuel equipment of automotive 
internal combustion engines. The working surfaces of the part are three flat faces. The part operates under difficult conditions of repeated cyclic loading in the presence of dirt, moisture and limited lubrication.

Two groups of parts with 10 experimental samples in each group were used for the research. The samples were subjected to all the necessary operations of the technological process of applying a wear-resistant anticorrosive diamond-like coating to the working surfaces - ultrasonic washing, applying a diamond-like coating in the DREVA 600 unit and polishing. The second group of samples after polishing was subjected to secondary coating in the DREVA 600 unit and polishing.

The surface of the coating was polished by mechanical-chemical-thermal method on a vertical milling machine $615 \mathrm{P}$. A steel end brush was used as a tool. The tool was fixed to the machine spindle instead of the cutter.

To determine the rational conditions for polishing, a complete three-factor experiment was performed on two levels. The range of variable factors is shown in table 1.

Table 1. Variable factors and areas of their determination.

\begin{tabular}{|c|c|c|c|c|c|}
\hline \multirow{2}{*}{ No } & \multirow{2}{*}{ Name of the factor, dimension } & \multirow{2}{*}{$\begin{array}{c}\text { Factors } \\
\text { designati } \\
\text { on }\end{array}$} & \multirow{2}{*}{$\begin{array}{l}\text { Code } \\
\text { mark }\end{array}$} & \multicolumn{2}{|c|}{$\begin{array}{l}\text { Boundaries of the } \\
\text { definition area }\end{array}$} \\
\hline & & & & Lower & Upper \\
\hline 1 & Tool rotation speed, $\min ^{-1}$ & $\mathrm{n}$ & $\mathrm{X} 1$ & 1000 & 2000 \\
\hline 2 & Cross feed, mm & $\mathrm{t}$ & $\mathrm{X} 2$ & 0.5 & 1.0 \\
\hline 3 & Longitudinal feed rate, $\mathrm{mm} / \mathrm{min}$ & S & $\mathrm{X} 3$ & 200 & 400 \\
\hline
\end{tabular}

The sample was fixed in a machine clamps. The cross feed of the tool was counted from the moment when the tool touched a paper sheet placed between the tool and the surface to be processed. $R a$ - the arithmetic mean deviation of the micro profile of the treated surface, microns, was considered as process indicators. At each point of the experiment plan, 3 samples were treated. Treatment was carried out in two working strokes - in the forward and reverse directions. The surface roughness after processing was measured with the Mar Surf PS1 device.

Linear dependences of the processing index on various factors were used as a mathematical model. Statistical processing of the experimental results and the construction of a regression dependence was carried out according to the generally accepted method. To test the hypothesis of homogeneity of variance, the Cochran criterion was used. The significance of the regression coefficients was determined by $t$ - the Student criterion. The adequacy of the model was evaluated by the Fisher criterion. In all these cases, the significance level was assumed to be $5 \%$.

Based on statistical processing of the experimental results, the following regression dependencies are obtained:

- for the working stroke in forward direction

- for the working stroke in reverse direction

$$
R a=0,1904+0,000059 n+0,0719 t+0,000202 S ;
$$

$$
R a=0,0838+0,000024 n+0,025 t+0,000072 S .
$$

Figure 1 shows the dependence of the arithmetic mean deviation of the micro profile of the treated surface on the speed of the tool after forward working stroke. 


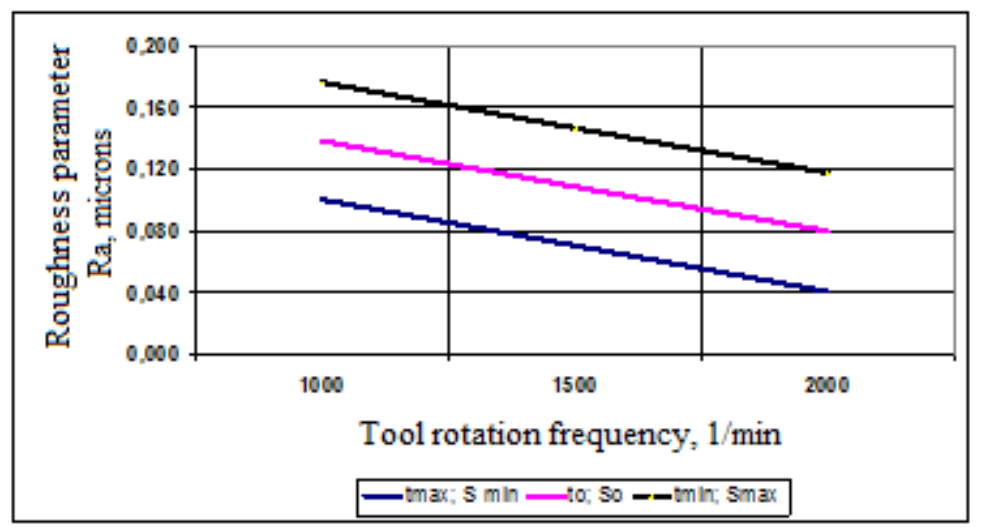

Fig. 1. Dependence of surface roughness $\mathrm{Ra}$, microns on the tool rotation speed $\mathrm{n}$ min ${ }^{-1}$ for various combinations of transverse $\mathrm{t}, \mathrm{mm} / \mathrm{stroke}$ and longitudinal feed rate $\mathrm{S}, \mathrm{mm} / \mathrm{min}$ after forward working stroke of the tool

Figure 2 shows the dependence of the arithmetic mean deviation of the micro profile of the treated surface on the speed of the tool after reverse working stroke.

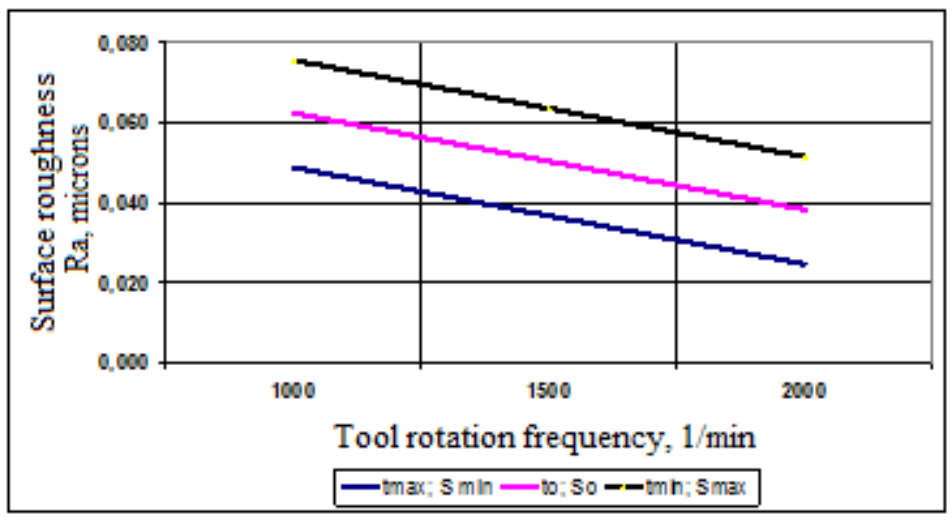

Fig. 2. Dependence of surface roughness $\mathrm{Ra}$, microns on the tool rotation speed $\mathrm{n} \mathrm{min}^{-1}$ for various combinations of transverse $\mathrm{t}, \mathrm{mm} /$ stroke and longitudinal feed rate $\mathrm{S}, \mathrm{mm} / \mathrm{min}$ after reverse working stroke of the tool

As it can be seen from figures 1 and 2, as the tool speed increases, the roughness of the treated surface decreases. This is explained by the fact that during the treatment process there are two interrelated factors - mechanical and chemical-thermal, and the effects of these two factors increase with increasing tool speed. The higher the speed of rotation of the tool, the greater force of the tool fires act on the tops of micro-roughness and ensure their chipping. In addition, the number of such strikes increases per unit of time. This leads to a reduction of surface roughness. The process of micro-chipping the tops of microroughness during the forward working stroke of the tool is so intense that the surface roughness decreases several times compared to the initial surface of the samples. Moreover, at the maximum value of the cross feed of the tool and the minimum longitudinal feed rate, this change occurs more significantly than at the minimum cross feed and the maximum longitudinal feed rate.

Although the mechanical factor at the first working stroke has a predominant influence, the friction energy increases with increasing tool speed and, as a result, the temperature in the treatment zone. This activates the chemical-thermal process, which also helps to reduce 
the surface roughness.

A comparison of figures 1 and 2 shows that the surface roughness after the second working stroke significantly decreased-by 1.7-2.3 times. The mechanism of this influence is that during the second working stroke, the influence on the surface roughness of the mechanical factor decreases, since the tool fibers slide on a smooth surface and the probability of micro-roughness chipping decreases. Therefore, during the second working stroke, with an increase in the tool rotation speed and, as a result, the temperature in the treatment zone, the intensity of the chemical-thermal process that occurs on the treated surface increases, as a result of which a phase transition occurs with the formation of graphite. In this situation, the bristles of the metal brush are a precursor to the graphitization process, and air oxygen is a reagent. The chemical-thermal process is more intense at the tops of micro-roughness than in the hollows, so there is a decrease in the roughness of very small micro-roughness left after chipping of large micro-roughness.

Figures 1 and 2 show that the value of the tool speed has a greater impact on the treatment result than other factors under study. Thus, if the tool speed increases by 2 times (Fig.2), this leads to a reduction in surface roughness by 1.5-2 times, then when the cross feed value is doubled, the reduction in surface roughness is from $20 \%$ at the minimum tool speed and maximum longitudinal feed rate to $80 \%$ at the maximum tool speed and minimum longitudinal feed rate. This effect of the longitudinal feed rate on the treatment result is explained by the fact that with increasing cross feed, the number of tool fibers in contact with the treated surface increases, and the contact area increases, since the fibers bend at a large depth and fit more closely to the treated surface. As the area of contact between the tool and the surface to be treated increases, the temperature in the treatment zone increases. All this activates the chemical and thermal process that occurs in the treatment area, and helps to reduce the surface roughness. But the value of the cross feed has little effect on the mechanical factor.

The longitudinal feed rate has the opposite effect to that which the tool rotation speed and the amount of its transverse feed have on the roughness of the treated surface. The surface roughness increases as the longitudinal feed rate increases. This is explained by the fact that with an increase in the longitudinal feed rate, the contact time of the tool with the treated surface decreases, and this leads to a decrease in the number of tool fibers hitting the protrusions of micro-roughness. In addition, as the longitudinal feed rate increases, the cooling conditions of the working area improve and the temperature in the treatment area decreases. The simultaneous reduction of both mechanical and chemicalthermal effects of the tool on the surface to be treated with an increase in the longitudinal feed rate increases the surface roughness.

Using regression equations (1) and (2), a method for optimizing treatment conditions was developed. The optimization criterion was the performance of the finishing process, which depends on the longitudinal feed rate. The roughness of the treated surface was a limiting factor. The required roughness of the trea ted surface is Ra 0.04 micron. However, the surface roughness values of the samples have a natural variation. Based on the experiment, the average square deviation of the surface roughness values for the reverse working stroke is obtained equal to $\sigma=0,0053$ microns. Therefore, the standard value of surface roughness is assumed to be $\mathrm{Ra}_{\mathrm{n}} 0,038$ microns. At this standard value of roughness, $90 \%$ of samples must have a surface roughness not exceeding the required one. The values of regime factors were limited by the experimental conditions shown in table 1.

Under these conditions, the optimal values of the polishing modes are as follows: the tool rotation speed is $2000 \mathrm{~min}^{-1}$; the longitudinal feed rate is $400 \mathrm{~mm} / \mathrm{min}$; the cross feed of the tool after it touches the treated surface is $0.5 \mathrm{~mm}$. Under these conditions, mechanical treatment of each of the above two groups of samples was carried out. The 
second group of samples was polished twice - after the first coating operation and after the second coating operation. All 10 samples installed on the machine table in a special device were treated at once.

Figure 3 shows the results of polishing of one of the sample groups as an example.

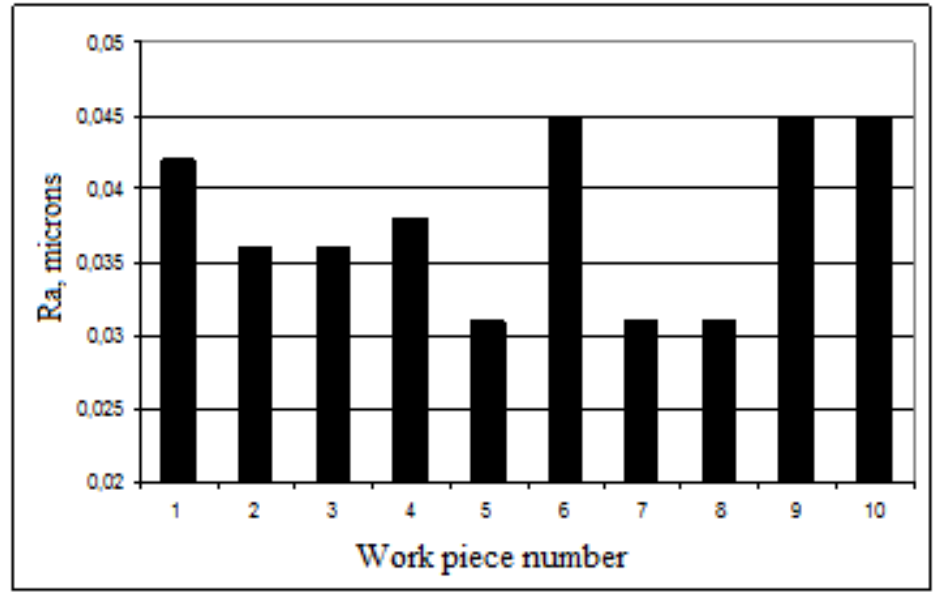

Fig. 3. Surface roughness of work pieces in a batch.

As it can be seen from figure 3 , the average surface roughness in this batch $\mathrm{Ra}=0,038$ is microns, $\sigma=0.0059$ microns. The proportion of samples with a surface roughness higher than the required one turned out to be slightly higher than the calculated one, but by an insignificant amount.

The structure of the coating surface was studied using an ultra-high-resolution scanning electron microscope Tescan Mira 2 LM. The microscope is equipped with a cathode with field emission and immersion optics. It is characterized by unsurpassed optical properties, non-flickering digital image of excellent quality. Modern, user-friendly software for microscope control, image accumulation and processing, running in a Windows ${ }^{\mathrm{TM}}$ environment. It has standard formats for saving images, a system for archiving, processing and convenient viewing of images, performing measurements on the image, an automatic system for configuring the microscope, and many other automated procedures. The study showed that the surface of the polished surface has a uniform structure, there are no metal build-ups and other foreign elements on the surface.

The depth of the coating was measured using an Olympus BX51 microscope by examining microsections made on a CALOTEST ${ }^{\circledR}$ DEVICE. Analysis of microsections of the treated surfaces of work pieces showed that the coating thickness is at least 2.1 microns, which is within the permissible limit. Therefore, it was decided to test the coating for corrosion resistance.

Corrosion tests were performed in a chamber of Ascott CC-450 equipment by exposing samples to salt mist with a water content of 2-3 g/cubic meter for 2 hours at a temperature of $(20 \pm 5)^{\circ} \mathrm{C}$. Then the temperature in the chamber increased to $(35 \pm 5)^{\circ} \mathrm{C}$, and the holding time was 22 hours. After that, the cycle was repeated. The total number of cycles of salt mist exposure to the samples was 7 , and the total duration of exposure was 168 hours.

After the tests were completed, a visual inspection of the test samples was performed. On the surface of images with a double ta-C coating, no changes on the surface and no traces of corrosion were found. All samples that had a single coating showed peeling of the coating and traces of corrosion on the surface under the 
coating. It follows that a double-applied protective layer of PVD coating ta-C protects the surface from corrosion.

\section{Conclusions}

Based on the performed research, it was found that the double ta-C coating of surfaces of precision parts by PVD method provides reliable anti-corrosion protection of the surface from moisture and aggressive media and can be used in the manufacture of agricultural machinery operating in difficult conditions of external influence.

\section{References}

1. O.Y. Filatov, V.I. Sidorko, S.V. Kovalev, Y.D. Filatov, A.G. Vetrov. Functional Materials, 1(23), 104 - 110 (2016)

2. J.M. Werrell, S. Mandal, E.L.H. Thomas, O.A. Williams, E.B. Brousseau, R. Lewis, P. Borri, P.R. Davies. Science and Technology of Advanced Materials, 1(18), 654-663 (2017)

3. E.V. Korostylev, V.S. Bormashov, S.A. Tarelkin, M.A. Doronin. Surface. X-ray, synchrotron, and neutron studies, 1, 74-78, (2017)

4. M.-L. Hicks, A.C. Pakpour-Tabrizi, R.B. Jackman. Diamond and Related Materials, (97), 107424 (2019)

5. A.V. Deryabkin. Electronics and electrical equipment of transport, 4, 35-37 (2018)

6. A.V. Deryabkin, Yu.Yu. Fedorov, M.P. Temiryazeva. Electronics and microelectronics of the UHF, (1), 550-551 (2018)

7. A. Kubota, S. Nagae, S. Motoyama, M. Touge. Diamond and Related Materials, 60, $75-80(2015)$

8. S. Roy, V.K. Balla, A.K. Mallik, N. Dandapat, V.G. Ralchenko, A.P. Bolshakov, E.E. Ashkinazi. A Comprehensive Study of Mechanical and Chemo-Mechanical Polishing of CVD Diamond, Materials Today: Proceedings, 9846-9854 (2018

9. Method of polishing the surface of polycrystalline diamonds: pat. RU 2369473. B24B $1 / 00(2009)$

10. Method of diamond plates polishing: pat. RU 2483856. B24B 37/04, B24B 1/00, 16 (2013) 